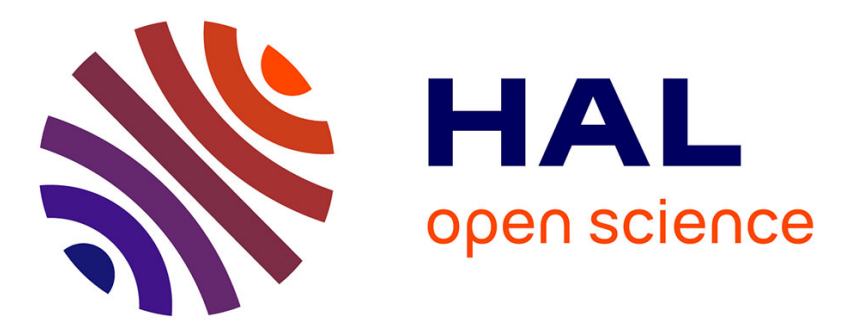

\title{
Industrial Implementation of Models for Joint Production and Maintenance Planning
}

\author{
Marco Macchi, Alessandro Pozzetti, Luca Fumagalli
}

\section{To cite this version:}

Marco Macchi, Alessandro Pozzetti, Luca Fumagalli. Industrial Implementation of Models for Joint Production and Maintenance Planning. IFIP International Conference on Advances in Production Management Systems (APMS), Sep 2014, Ajaccio, France. pp.499-506, 10.1007/978-3-662-447390_61. hal-01388584

\author{
HAL Id: hal-01388584 \\ https://hal.inria.fr/hal-01388584
}

Submitted on 27 Oct 2016

HAL is a multi-disciplinary open access archive for the deposit and dissemination of scientific research documents, whether they are published or not. The documents may come from teaching and research institutions in France or abroad, or from public or private research centers.
L'archive ouverte pluridisciplinaire HAL, est destinée au dépôt et à la diffusion de documents scientifiques de niveau recherche, publiés ou non, émanant des établissements d'enseignement et de recherche français ou étrangers, des laboratoires publics ou privés. 


\title{
Industrial implementation of models for joint production and maintenance planning
}

\author{
Marco Macchi ${ }^{1}$, Alessandro Pozzetti ${ }^{1}$, Luca Fumagalli ${ }^{1}$ \\ ${ }^{1}$ Department of Management, Economics and Industrial Engineering, Politecnico di Milano, \\ Piazza Leonardo da Vinci 32, 20133 Milano. \\ \{marco.macchi, alessandro.pozzetti, luca1.fumagalli\}@polimi.it
}

\begin{abstract}
The paper aims at investigating the industrial applicability of models for joint production and maintenance planning. Scientific community has paid attention to this issue for decades and more recently many researchers proposed models to optimize such kind of planning. Nevertheless each scientific work is based on its peculiarities and hypothesis, which might prevent the applicability within different industrial contexts. This is also due to the very theoretical roots of many models, often tested only within numeric or simulation scenarios. This paper aims at presenting a case study analysis where the industrial test-bench is analysed to understand how these types of model are adaptable and extendable to a real context. To support the case study analysis, the main literature on the topic is reviewed, with the purpose to provide the background for the model deployment and test within the selected industrial context.
\end{abstract}

Keywords: Production Planning, Maintenance planning, Economic Production Quantity (EPQ), Manufacturing Industry.

\section{Introduction}

Production and maintenance management are responsibilities of different functions often corresponding to different departments of a manufacturing company. This could lead to an un-optimized management that does not seek for the overall maximization of operations performance, but only of production and maintenance. Conversely, a joint vision would allow to get an optimized management, in coherence with the single objectives of the two departments, but also aligned with the overall goal of the company. In this regard, all practices, rules and, in general, models that allow a joint and coordinated management are herein considered as "models" for joint production and maintenance planning in different time horizons; further on, within this research, a specific concern is given to mathematical models for optimization.

Considering these models, it is worth pointing out that in literature many works are dedicated to the optimization of production and the optimization of maintenance, but a practical point of view on a joint approach is not so diffused. Recently, models have been distinguished based on the time horizon they refer to in their planning scope. In particular, according to [1], models are used for the (i) long term strategic and maintenance concept, (ii) medium term planning (i.e. tactical level) and (iii) short 
term scheduling; besides, another category of scientific works is specifically focused on control and performance indicators.

This paper deals with models supporting decisions at tactical level and it is based on a case study. The case allows studying the industrial applicability of such kind of models within a real context. This is aligned with the long-term objective of our research: contributing to fulfill the gap between the scientific theories and industrial practices with regard to the joint production and maintenance management. After a brief literature review (section 2), the model developed for industrial applicability is presented with related testing cases (section 3 ) and then used within a manufacturing industry case study (section 4); conclusions are eventually provided (section 5).

\section{Literature Review}

\subsection{Brief state of the art analysis}

Literature background is mostly based on two papers which revise the state of the art [1,2], presenting a classification of models for aggregate planning and scheduling.

According to [1], a first group of models - strategic decision models - is used during the system design phase, in order to identify at a high level the type of maintenance policy that should be applied in view of the production requirements of the designed system. The second group of models - tactical decision models - is focused on decisions over a mid-term (i.e. between one month and one year as time horizon). Such models support decisions for important maintenance interventions, deciding whether and when they should be performed based on their impact on the production plan for what concern the delivery of the demand of the planned period; this kind of models often considers the finite capacity of the maintenance crew in terms of hours that they make available for the activities of the maintenance plan. The last group of models - short term scheduling - considers a short time horizon, in order to schedule the interventions on lines or on singles machine based on an elaborated plan; this type of models takes care of deciding when a planned intervention must be scheduled within (often) one day timeframe.

[2] propose to analyze the scientific works by means of three areas of interest: Quality, Maintenance, Production. The authors then divide the works based on how these areas of interest are considered, by a joint approach, within the mathematical models provided by the papers surveyed from literature. Focusing on the relationships between two areas, Production and Quality are worth to be firstly discussed. They are intrinsically linked, considering that a production process is imperfect. This implies that scraps and WIPs are generated in an un-optimized manner, thus economically impacting on the performances of the production process. A quality factor is then included in models for the Economic Production Quantity (EPQ). These models are a variant of models for Economic Order Quantity (EOQ), defined when a production process is considered (instead of acquisition process). The imperfection of the process is generally modelled by its deterioration as a function of time with, for instance, a linear or exponential model. Quality problems might be then detected by inspections, often considered by the models, including their cost. This approach allows to optimize the EPQ and the inspection interval to minimize the overall costs. Instead, for what 
concern the joint approach on Production and Maintenance areas, the main highlight concerns the relationship between production and maintenance in planning, often seen by separated approaches, pursuing an optimal maintenance plan, given the production plan or vice-versa. Also in this case, the EPQ models are used, considering the tradeoff between preventive maintenance cost and the cost associated to the risk of corrective maintenance during the production period (in some cases also including the risk of defective products, that is the lack of quality). Further on, still within the relationship between Production and Maintenance areas, it is worth considering that the negative effect of stoppages might be also mitigated by the material kept on stock, which can be used to supply a given demand when production is not possible: in this regard, a good number of models considering the production systems with buffer capacity is proposed in literature. Eventually, the Maintenance and Quality areas are also sometime jointly addressed while neglecting the Production area. This is done basing on typical approaches as the following ones: a study of how maintenance, as an imperfect activity, impact on quality; an orientation towards using maintenance to reduce the quality gap with respect to a target value.

\subsection{Selection of reference models from literature}

Starting from the literature surveys of $[1,2,3]$, their references have been considered for more in depth analysis (respectively, [1] cited 88 papers, [2] 116 papers, [3] 45 papers). The references, integrated with other papers published during the more recent years, allowed us to select a sample of 90 papers as background for our research. Firstly, the analysis of this sample allows to assert that not all the scientific works provide a validation or verification of the proposed model. Moreover, only few of the sampled papers verify the models by means of an application in real world (6 papers, out of 90 papers of the sample), while the large amount of works considers validation only through numeric example (59 out of 90 ) or simulation (15 out of 90 ). This result further justifies our intention to concentrate on industrial applicability. Moreover, the objective of this analysis was to identify some reference models to be used for the definition of a model candidate to be tested in the industrial case study. Thus, as the 90 papers present optimization models for joint maintenance and production planning and scheduling, models were initially classified according to 7 clusters. The clusters are primarily inspired by the classification of [1]. Amongst them, 3 clusters are grouping the tactical models, as main concern of our research: EPQ models with quality issues; EPQ models with failure issues, Models considering systems with buffer capacity. EPQ with failure issues is the cluster of models this research has been concentrating on: more than a quarter of papers in our sample presents EPQ models with failure issues; based on this simple statistic, these models can be reasonably considered as the most addressed by the scientific community.

Thus, focusing only on the EPQ models with failure issues, we identified some decision drivers in order to fine-tune the selection of models. The linearity and easy understandability of the models have been considered as relevant properties for the industrial application, assuming that they would enable a more transparent approach to the industrial user. Moreover, production and maintenance have been focused as main target, not neglecting necessarily models that consider also quality as third area covered by the model. Eventually, the type of production system assumed by the 
model was also taken into account, in order to select the joint planning models that apply on properly detailed systems, thus at least comparable to real industrial systems.

\section{Deployment of the proposed model}

\subsection{Overview of the proposed model}

Literature analysis led to select some of the most recent models: [4,5]. [4] propose a model oriented to follow a given production optimization. The maintenance plan is subordinated to a strict constraint: the realization of the entire demand of each type of product. Backlog is not considered and demand must be strictly respected. The model considers a series of machines (a production line), introducing flexibility to represent both the system as a whole and the single phases and stations. Finally, each machine is subject to a Preventive Maintenance (PM) only once within the production planning bucket. [4] has been selected as good reference of a model that properly takes care of production priorities. [5] propose a model oriented to follow a given maintenance plan. Optimal PM frequency is then fixed and must be respected. Based on a certain maintenance behavior of the system, represented through failures' models, the model optimizes the production plan of orders, provided within a given time window (i.e. defined by the earliest and latest time for production). Backlog is considered through the related backorder costs; also overtime, if needed, can be exploited. Finally, the system is represented as a whole, even if failures' models provides a good lever for detailed modelling of the system. [5] was selected as good reference of a model that give adequate priority to maintenance plan. The two models are, thus, representative of two different attitudes, that is priority to production and priority to maintenance. Both models then reveal pros and contra. [4] model is good for a well deployment of the production system model by considering the machines / subsystems, while it lacks in a detailed modelling for overtime work and flexibility in the number of PM within a production time (only one PM is allowed). [5] model, instead, is good for its modelling capability of overtime work, but does not allow decomposition of the system and represents PM as fixed in its scheduling. Hence, this research aimed at the preparation of a new model as a fusion of the features provided by the two models. Hence, based on their pros and contra, requirements for a new model were firstly elicited. Requirements come from the need to support a decision maker within a manufacturing industry with a good flexibility to cope with both production and maintenance planning issues. A company producing white-goods offered a test-bench to this end. The company operates a mass-production. The production system of the test-bench is a manufacturing line; therefore, the system reliability is in accordance to a series logic: a stoppage of one of the machines in the line implies the stoppage of the entire line. This strongly requires an accurate maintenance planning, coordinated with production, to optimize availability of the line with the purpose to guarantee the production targets. Further details of the application of the model in the case study are proposed in section 4 . Then, the following are the requirements identified for the deployment of a new model: i) frequency of PM is not fixed a-priori; ii) one PM always occur at the first period in order to restore the system at an "As-Good-AsNew" state; iii) PM can occur more than once (generally twice) within the production 
time horizon; iv) the production system is considered divided in sub-systems, like machines, for quantifying the maintenance performances; v) the production system is considered as a whole for the production performance; vi) backlog is allowed; vii) overtime work is also exploitable. Overall, according to the requirements, the [4]'s model has been extended by the introduction of two decision variables - overtime work and backlog - and some new specific constraints (derived from [5]).

\subsection{Testing scenarios}

An experimental plan was defined for a numerical validation of the developed model. From the literature, considering the typical variables involved in the analysis, the followings have been considered for the plan: production mix variability, demand predictability, aggregate planning strategy. Each variable can be considered with two levels: high (H) or low (L) value for variability and predictability, Level or Chase for the planning strategy. Thus, with the two levels and three variables, $2^{3}$ scenarios are possible. Nevertheless, some key scenarios have been considered, out of all possible scenarios, envisioning their meaningfulness in some real industry. This led to identify three experimental conditions: i) Level strategy, high demand predictability, low mix variability; ii) Chase strategy, high demand predictability, low mix variability; iii) Chase strategy, low demand predictability, high mix variability. These scenarios were then created with numerical examples and applied to the proposed model, after representing the uncertainty (subsequent to demand predictability) based on Monte Carlo simulation. The numerical examples helped validating the new model, which has achieved similar or even better performances than the original models of [4,5].

Indeed, the case study corresponds to one of the scenarios used for numerical testing, namely the first one featuring a Level strategy, high demand predictability and low variability of the production mix (like working under conditions of a "quasiconstant" mix, with a low uncertainty for what concern the demand prediction). In this scenario, it has been seen that stocking material is a solution to prefer than requiring overtime work. To this end, the model proposed by [5] is not able to flexibly manage the preventive maintenance scheduling and result in a schedule that cut considerably available working time, forcing than to consider overtime working (even in a scenario where nominal capacity can satisfy a stable demand). The new model, instead, manage the risk of possible stoppages, balancing such risk, creating a scheduling that more reasonably consider the exploitation of the production capacity of the system. The new model was, thus, considered ready for an application in industry and judged as good reference in order to further work on a test in a real case.

\section{Manufacturing industry case study: analysis and results}

By applying the model to the case the results theoretically achieved are equal to an overall saving of $57 \%$ of maintenance costs, compared with the AS-IS situation. This seemed a very interesting result, considering that in literature models for optimization of maintenance and production scheduling are claimed to be able to provide a saving of around 30\% [6]. Starting from this potential, the analysis of the case was further 
directed to critically understand the reasons, identifying the hypothesis laying behind this behavior. Indeed, what is worth to be herein discussed is the sensitivity analysis, performed on the model with the purpose to check how it behaves. The methodology for case study analysis, that has been thus applied, followed some steps related with sensitivity analysis: i) the results given by the model have been compared with the actual practices of the company, ii) great differences between what suggested by the model and what presently ongoing as practices in the company were carefully checked, iii) the weaknesses of the model, namely some restrictive hypothesis not originally identified, were highlighted as a final outcome.

\subsection{Analysis on production capacity variation}

First variable checked by sensitivity is production capacity. Production capacity has been intended by the company as a reduced time available for operations (shortly, in the remainder, operating time), resulting from either a reduction of shifts or closure days. This was compliant with the hypothesis of use of the model.

As a general consideration on the model behavior, it has been observed that the production capacity results correlated with production system utilization: a decrease of production capacity corresponds to an almost linear increase of utilization. Further on, the proposed model considers the PMs scheduled within operating time; their further impact should be then measured: subsequently, when decreasing production capacity, it has been noted that the model suddenly proposes to use overtime work in order to guarantee the demand; on the other hand, the variation of production capacity does not show any correlation with the plan of PMs suggested by the model, that remains unchanged. This can be explained basically considering the ratios of costs adopted for experimentation: the overtime work is costing less than backlogs and PMs; then, the model shows a capability to adapt its planning strategy based on cost minimization. In other words, in order to counterbalance the decreasing capacity, the overtime work is the resource used because is the less costing.

In comparison with the actual practices of the company, what suggested by the model was instead judged as not properly aligned. In fact, the practice of the company is to try to avoid the overtime work when the nominal production capacity is reduced and, instead, to neglect some PM interventions in comparison with the original plan, in order to save some operating time for production. This has been proved, based on experience over the years, not impacting on availability of the line. After discussing such a behavior, the root cause of misalignment of the model with the real conditions was identified: the reliability of the machines is in fact considered by the model as independent from the production capacity, hence the operating time of the line. More precisely, our model followed what normally proposed in literature: to consider the reliability only as a function of the calendar time. But reliability is also a function of the type of use, hence the operating time and other minor variables, especially true with electromechanical systems, as the machines of the line, that should be normally assumed behaving under so called Operating Dependent Failure (ODF). Thus, the case study highlighted how the type of use and the operating time should not be neglected, but instead should be taken into account by the model in order to represent a reliability behavior that is influenced by the capacity that the system deploys for achieving the production targets. 


\subsection{Analysis on maintenance parameters variation}

Maintenance parameters, time and costs, are the second type of variables checked by sensitivity analysis, to verify their effect on the comprehensive cost of the plan, and the number of maintenance interventions. In this regard, it is worth underlining that the failure distribution model adopted for the case is a Weibull function, according to well-known reliability theory, while the parameters of the Weibull have been derived based on an analysis of the historical data recorded in the company's maintenance information system at machine level. Considering an increase of cost of Corrective Maintenance (CM) interventions or of time needed to fix problems (equivalent to say that failures become more critical events), the model pushes to carrying on more PMs. More specifically, this trend is observed for the machines of the production system whose occurrence of failure is modelled by a Weibull with a form factor larger than 1 , representing the behavior in a wear-out phase of the bathtub curve, well-known in the reliability theory. Conversely, for other machines, where the form factor is equal to 1 (i.e. the failure occurs based on a memory-less phenomenon, modelled by an exponential distribution), the model does not suggest PM, that practically would be not necessary, being the failures not depending on the elapsing of time. Eventually, when the form factor of the Weibull is minor than 1 , the model leads to reduce as much as possible the number of PMs, that has not positive impact on the reduction of failures. All in all, the behavior observed with different Weibull models, changing based on different form factors, is aligned with expectations from reliability theory, in particular, with literature on maintenance cost optimization models.

Nevertheless, a misalignment with actual practices of the company was detected, in the cases of Weibull with form factor minor than 1. Further investigation then aimed at checking whether practices of the company might be considered wrong, or if some data introduced in the model were not aligned with the actual hypothesis. Indeed, the machines whose failures are characterized by a Weibull distribution with form factor minor than 1 are currently maintained with much more PMs than what suggested by the model. The mathematical formulation behind our optimization model, instead, considers that for these machines it is not worth carrying out PMs. In particular, according to how the model behaves based on its laying assumptions, the machines are considered "As-Good-As-New" after each PM and, in this case, they would start to suffer of more failures due to the case of infant mortality (i.e. the wear-in phase of the bathtub curve, correspondent to a form factor of the Weibull function minor than 1). According to this behavior, the model thus suggests to carry out less PMs than the ones presently carried out in the company. Nevertheless, this is a blind application of the mathematical model that risks to create a bias and even errors in maintenance planning. The most reasonable root cause of the error is using the Weibull analysis at a machine level. This hides the different failure modes that the machine can suffer from, and how the PMs contrast such failure modes. In effect, in reality, not all the PMs are an overhaul intervention on the machine; instead, they are of different types (e.g. minor regulations, cleaning, etc...) and address only few failure modes, thus being far from the hypothesis of returning the machines to an "As-Good-As-New" condition. Therefore, the model certainly shows one of its drawback when the overall behavior of the machine is modelled with Weibull with factor form $<1$, while some 
PMs are carried out just to contrast few failure modes that, at a detailed level, could result modelled by a Weibull with factor form $>1$. Making a detailed modelling of behavior of the machine is well discussed in reliability analysis, and, from our result, it seems worth of consideration within models for joint production and maintenance planning. Indeed, by the herein presented case study, it has been verified the strong impact it can have on the industrial usability of such kind of optimization model.

\section{Conclusions}

The paper introduced the topic of joint production and maintenance planning and scheduling by summarizing three main surveys in the research area. A new model based on existing scientific works has been presented by means of a brief explanation, neglecting the mathematical formulation, out of the scope of the present dissertation while focusing on its use in an industrial application. Indeed, the industrial application revealed some quite relevant differences between the recommendations resulting from the model and the actual practices carried on by the company. This has allowed to highlight some weaknesses of such kind of models, that we deem quite interesting in terms of possible future research.

On the whole, the analysis revealed how the impact of variance or uncertainty of inputs related with maintenance issues can be quite serious, possibly more relevant than the uncertainties due to production inputs. Unfortunately, the maintenance data are the ones that are often less considered, hence less modelled, in the companies. The case study then arises a warning on a proper management of maintenance data and, in particular, on maintenance performance, which can be really affecting overall plant performance and, more specifically, a coordinated production and maintenance plan.

Acknowledgements. The authors would like to thank Alessandra Del Centina for her support in the research through the deployment of her master of science thesis.

\section{References}

1. Budai, G., Dekker R., Nicolai R.P.: Maintenance and Production: A Review of Planning Models. In Complex System Maintenance Handbook, 321-344. Springer London (2002)

2. Ben-Daya M., Rahim M.: Integrated production, quality \& maintenance models: an overview. In M. Rahim and M. Ben-Daya (eds), Integrated models in production planning, inventory, quality, and maintenance, Kluwer Academic Publishers, 3-28 (2001)

3. Schimdt, G.: Scheduling with limited machine availability. European Journal of Operational Research,121: 1-15 (2000)

4. Sitompul, C., Aghezzaf E. H.: An integrated hierarchical production and maintenanceplanning model. Journal of Quality in Maintenance Engineering, 17(3): 299-314 (2011)

5. Najid, N. M, Alaoui-selsouli M., Mohafid N.: An integrated production and maintenance planning model with time windows and shortage cost. International Journal of Production, 49: 2265-2283 (2011)

6. Cassady, C.R., E. Kutanoglu: Integrating Preventive Maintenance Planning and Production Scheduling for a Single Machine. IEEE Transactions on Reliability, 54 (2):304-309 (2005) 